\title{
INFLUENCIA DE EL NIÑO EN EL PAÍS VASCO
}

\author{
Maialen Martija-Díez ${ }^{(1,2,3)}$, Belén Rodríguez-Fonseca ${ }^{(3,4)}$ \\ (1) Tecnalia R\&I (Área Meteorología), maialen.martija@tecnalia.com \\ (2) Agencia Vasca de Meteorología (Euskalmet) \\ (3) Universidad Complutense de Madrid, Dept. de Geofísica y Meteorología \\ ${ }^{(4)}$ Instituto de Geociencas IGEO, CSIC UCM
}

\section{Introducción}

La variabilidad climática en Europa ha sido profundamente analizada y se ha relacionado tanto con la variabilidad interna de la atmósfera como con el forzamiento que origina la temperatura de la superficie del océano, incluso a miles de kilómetros (teleconexiones). Centrándonos en la influencia del océano Pacífico Tropical sobre la variabilidad climática en Europa, numerosos estudios concluyen que el impacto de ENSO (El Niño Sourthern Oscillation) en el área euro-atlántica es robusta y estadísticamente significativa, a pesar de su debilidad (Fraedrich, 1994). Algunos estudios se centran en la influencia de El Niño sobre la precipitación en Europa (Bulić \& Kucharski, 2012; Raible et al., 2003; Stockdale et al., 1998), y otros, en cambio, analizan la influencia de este fenómeno sobre la temperatura (Bergman, 1984; Kiladis \& Diaz, 1989). Estudios recientes ahondan en cómo diferentes patrones espaciales de ENSO producen distintas respuestas en Europa y profundizan en la explicación de estas diferencias centrándose en tipos de teleconexiones distintas (Zhang et al., 2018). Por un lado, se describen las teleconexiones tipo circumglobales (CGT), ondas que quedan atrapadas en el jet del norte y que presentan una estructura con 5 centros negativos y otros 5 positivos (número de onda 5) (Branstator, 2002; Ding \& Wang, 2005). Por otro, las teleconexiones de tipo arco, que se caracterizan por aprovechar un debilitamiento del jet para atravesarlo. Estas últimas pueden ser del tipo Pacific North American (PNA) o Tropical Northern Hemisphere (TNH) (Barnston et al., 1991; Mo \& Livezey, 1986) y Yu et al. (2015) encuentran como la PNA aparece en los casos en los que el patrón espacial de ENSO es de tipo central o del este, mientras que la TNH se relaciona con Niños del este.

La mayoría de los estudios analizan la influencia de El Niño en Europa en el invierno boreal, momento en el que se registra el pico de este fenómeno, pero Ding et al., (2011) profundizan en la posible influencia en el verano anterior, cuando el fenómeno comienza a desarrollarse, y el posterior, cuando este va debilitándose. Estos autores concluyen que en los veranos anteriores al pico de El Niño la teleconexión que aparece sigue un patrón CGT, mientras que en los veranos posteriores al fenómeno el patrón es tipo PNA.

Sin embargo, también se ha observado que la influencia de ENSO sobre Europa no es lineal, de manera que la influencia de La Niña no es la contraria que la de El Niño (Cassou \& Terray, 2001; Davies et al., 1997; Dommenget et al., 2013; Hannachi, 2001; Toniazzo \& Scaife, 2006; Wu \& Hsieh, 2004a, 2004b), y esta respuesta tampoco es estacionaria en el tiempo, estando modulada a escalas mutidecadales (Brönnimann, 2007; LópezParages \& Rodríguez-Fonseca, 2012; López-Parages et al., 2015, 2016). Algunos autores sugieren que la teleconexión entre ENSO y Europa viene modulada por la temperatura del océano Atlántico (Gouirand \& Moron, 2003; Mathieu et al., 2004; Pozo-Vázquez et 
al., 2001; Sutton \& Hodson, 2003), sin embargo estudios recientes señalan que la Atlantic Multidecadal Oscillation (AMO) y la Pacific Decadal Oscillation (PDO) (López-Parages \& Rodríguez-Fonseca, 2012; López-Parages et al., 2015, 2016; Zanchettin et al., 2008; Zhang et al., 2012) modulan esta teleconexión.

En este estudio se ha elegido la zona del País Vasco (PV) para analizar la influencia que tiene el fenómeno de El Niño sobre la temperatura máxima (Tmax), la temperatura mínima (Tmin) y la precipitación (Pcp) en la región. Esta zona está situada en el norte de la península Ibérica (PI), y tiene unas características climáticas diferentes a gran parte del resto de España. Mientras que la mayor parte del país tiene una estación seca, la zona norte (Biau et al., 1999; Mills, 1995; Serrano et al., 1999; Zorita et al., 1992), aunque menor cantidad, registra lluvia también en verano. Sáenz et al. (2001) analizaron la influencia de la North Atlantic Oscillation (NAO) y también de la Arctic Oscillation (AO) sobre la zona norte de la PI, pero no la de ENSO. En este trabajo, nos hemos centrado en el análisis de la influencia de ENSO sobre la Tmax, Tmin y Pcp en las 12 posibles estaciones de 3 meses del año, desde enero-febrero-marzo (JFM) hasta diciembre-enero-febrero (DJF). También hemos profundizado en los mecanismos que explican la teleconexión entre El Niño y la variabilidad climática en el PV en verano.

\section{Metodología}

En este trabajo se ha utilizado Spain02_v2.1 (Herrera et al., 2012), datos de rejilla de alta resolución $\left(0,2^{\circ} \times 0,2^{\circ}\right)$ sobre España para los datos de Tmax, Tmin y Pcp. La región elegida ha sido, $42,2^{\circ} \mathrm{N}-43,4^{\circ} \mathrm{Ny} 3,6^{\circ} \mathrm{W}-1,6^{\circ} \mathrm{W}$. Los datos de las variables atmosféricas utilizados vienen del reanálisis NCEP/NCAR (Kalnay et al., 1996), con una resolución de $2^{\circ} \times 2^{\circ}$. En particular se han utilizado datos de temperatura a $850 \mathrm{hPa}$ (T850), presión a nivel del mar (SLP) y altura geopotencial a $500 \mathrm{hPa}$ (Z500) sobre sobre la zona del Atlántico Norte $\left\{20^{\circ} \mathrm{N}-60^{\circ} \mathrm{N}, 50^{\circ} \mathrm{W}-30^{\circ} \mathrm{E}\right\}$, y altura geopotencial y velocidad zonal de viento a $200 \mathrm{hPa}\left(\mathrm{Z} 200\right.$ y U200) en la banda tropical y en el hemisferio norte $\left\{30^{\circ} \mathrm{S}-90^{\circ} \mathrm{N}\right.$ and 0 $\left.{ }^{\circ} \mathrm{E}-360^{\circ} \mathrm{E}\right\}$. Finalmente, la temperatura en la superficie del mar (SST) se ha obtenido de la Hadley Centre Sea Ice and Sea Surface Temperature dataset (HadISST, Rayner et al., 2003), con una resolución de $1^{\circ} \times 1^{\circ}$. Con estos datos se calculan los índices de El Niño definidos como las anomalías en diferentes cajas en el Pacífico Tropical; El Niño $1.2\left\{10^{\circ} \mathrm{S}-0,90^{\circ} \mathrm{W}\right.$ $\left.-80^{\circ} \mathrm{W}\right\}$ y El Niño $4\left\{5^{\circ} \mathrm{S}-5^{\circ} \mathrm{N}, 170^{\circ} \mathrm{W}-120^{\circ} \mathrm{W}\right\}$.

El periodo utilizado para todas las bases de datos va desde 1950 hasta 2007, analizando las 12 posibles estaciones de 3 meses del año, desde JFM de 1950 hasta diciembre 2006-enero-febrero 2007. La variabilidad de la Tmax, Tmin y la Pcp se analiza calculando las anomalías estacionales para cada año, restando a la media anual la media de todo el periodo. Por otro lado, para analizar la variabilidad interanual se le aplica a la serie un filtro Butterworth con la frecuencia de corte de 7 años. Después, con el objetivo de encontrar periodos estacionarios en la relación entre ENSO y la variabilidad en las variables analizadas (Tmax, Tmin y Pcp) sobre el País Vasco, se calculan las correlaciones entre dichas variables y los índices de El Niño en ventanas corridas de 20 años, es decir, para JFM partiendo del periodo 1950-1969 y terminado con 1987-2006 (siguiendo la metodología de López-Parages \& Rodríguez-Fonseca, 2012). 
Centrándonos en los periodos con correlación significativa, analizamos la teleconexión que explica esta relación. Para ello, se computan mapas de regresión horizontal y correlación, proyectando (correlacionando) los índices de la Tmax, Tmin y la Pcp sobre los campos anómalos de la SST, Z200 y SLP, añadiendo el campo anómalo de Z500 para la Pcp y el de T850 para Tmax y Tmin.

Para profundizar en el comportamiento no lineal de la teleconexión entre ENSO y la variabilidad climática en Europa (Brönnimann, 2007), se han creado mapas de compuestos positivos y negativos de las variables, para analizar la influencia de El Niño y La Niña sobre el PV por separado.

Finalmente, para evaluar la posible predictibilidad del fenómeno y analizar su evolución, se calculan correlaciones entre las variables en las estaciones y periodos seleccionados y los índices de El Niño en estaciones anteriores, hasta 2 años atrás en total.

A lo largo de todo el estudio, se utilizan test de significación estadística al 95\%; test de correlación, test de diferencia de medias (Student's t-Test) y de desviación estándar (Fisher's F-Test).

\section{Resultados}

La influencia de ENSO sobre la variabilidad de Tmax, Tmin y Pcp en el PV se analiza realizando correlaciones móviles en ventanas de 20 años entre dichas variables y los índices de El Niño (El Niño 1.2 y El Niño 4) en las 12 posibles estaciones de 3 meses del año. Los resultados muestran una relación no estacionaria para todas las variables (Fig.1), en concordancia con López-Parages \& Rodríguez-Fonseca (2012) y CapaMorocho et al. (2014).

Correlaciones altas y significativas aparecen en todas las variables analizadas y para los dos índices de El Niño analizados, en varias estaciones y décadas. Pero sorprendentemente las correlaciones más altas aparecen en verano (julio-agosto-septiembre; JAS) tanto para la Tmax, Tmin como para la Pcp. Por lo tanto, a pesar de que aparecen correlaciones significativas en otras estaciones del año, considerando que la mayoría de los estudios anteriores se centran en invierno, primavera u otoño, hemos elegido únicamente los periodos de mayor correlación en verano para realizar un análisis en profundidad (Tabla 1 y cajas negras en la Fig. 1).

$\begin{array}{lcc}\text { VARIABLE } & \text { ESTACION SELECCIONADA } & \text { PERIODO SELECCIONADO } \\ \text { Tmax } & \text { JAS } & 1952-1987 \\ \text { Tmin } & \text { JAS } & 1952-1987 \\ \text { Pcp } & \text { JAS } & 1957-2003\end{array}$

Tabla 1.- Periodos con mayor correlación entre las variables y los índices de El Niño, seleccionados para realizar el estudio.

En las Fig. 2 y 3 se muestran los mapas de regresión de las anomalías estandarizadas de Tmax, Tmin y Pcp, sobre los diferentes campos de las variables atmosféricas anómalas. Analizando la regresión sobre la SST (Fig. 2), encontramos una señal muy parecida para Tmax (arriba) y Tmin (medio) en el Pacífico, aunque más débil en el caso de la 
segunda. Aparece un patrón negativo, lo que indica que los años con anomalías positivas en Tmax y Tmin, se pueden relacionar con La Niña (ENSO negativo). Por construcción, los años con anomalías negativas en Tmax y Tmin, se relacionan con ENSO positivo. El patrón de El Niño (La Niña) que se obtiene está en ambos casos centrado en el Pacífico Ecuatorial, pero los valores de la SST son mayores en el caso de la Tmax, $-0,5^{\circ}$ en varias zonas, frente a los $-0,2^{\circ}$ que aparecen en el caso de la Tmin. Dado que las variables han sido estandarizadas, podemos concluir que el océano necesita modificar menos su temperatura para producir la misma variabilidad en la Tmin que en la Tmax en el PV.
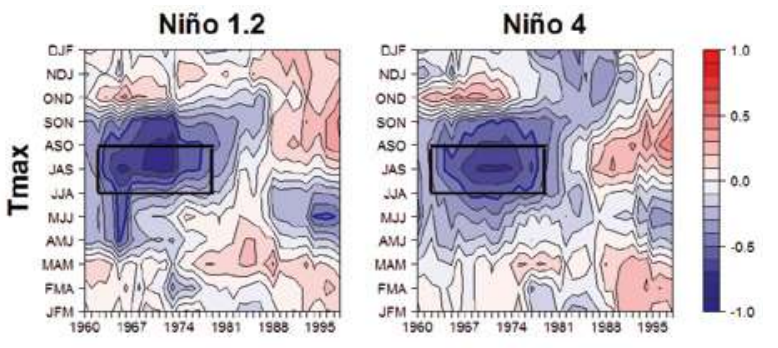

Fig. 1.- Correlaciones móviles en ventanas centradas de 20 años en el PV entre: Tmax (arriba), Tmin (centro) y Pcp (abajo) y el índice de El Niño 1.2 (izquierda) y El Niño 4 (derecha) en las 12 posibles estaciones trimestrales del
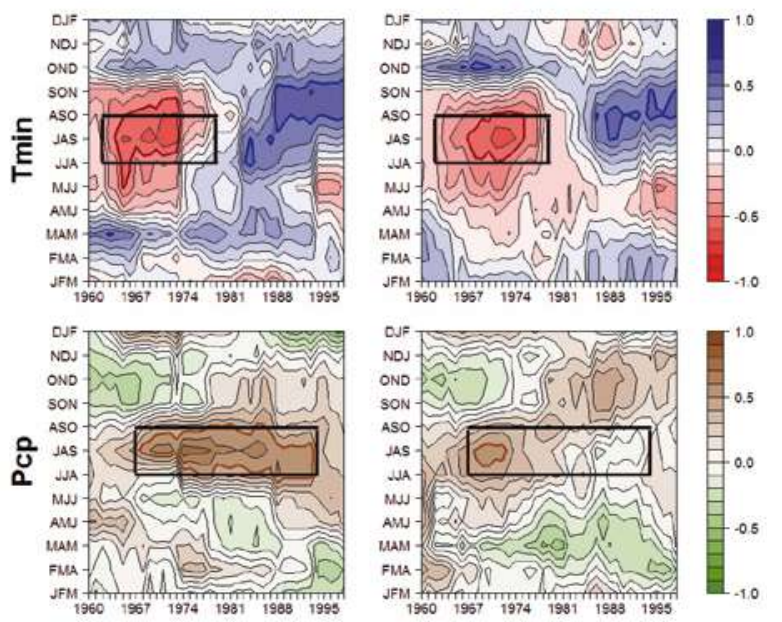
año. Líneas coloreadas para correlaciones estadísticamente significativas.

El patrón en las anomalías de Z200 en Europa, para el caso de Tmax y Tmin (Fig. 2, arriba y medio, contornos), muestra una estructura en la teleconexión que regionalmente está caracterizada por un centro anómalo positivo en Francia, que abarca también la PI y el PV. Este centro se puede relacionar con un aumento en los eventos de bloqueo de la circulación general oeste-este, que puede, por lo tanto, aumentar la estabilidad atmosférica en la zona. Esta configuración tiene también su reflejo en superficie (Fig. 3, izquierda y medio, contornos), donde aparece un centro de anomalías positivas en la SLP sobre Francia, cubriendo el oeste de Europa, incluyendo el PV. Más aún, la posición concreta de este centro indica un aumento de la frecuencia de vientos del sur, que se puede relacionar con un aumento de la temperatura en la zona. En el mapa de regresión de la T850 (Fig. 3, izquierda y medio, en colores), aparece esta anomalía positiva, superando $0,6^{\circ}$ en el PV para el caso de la Tmax, y acercándose a los $0,5^{\circ}$ para las Tmin. 

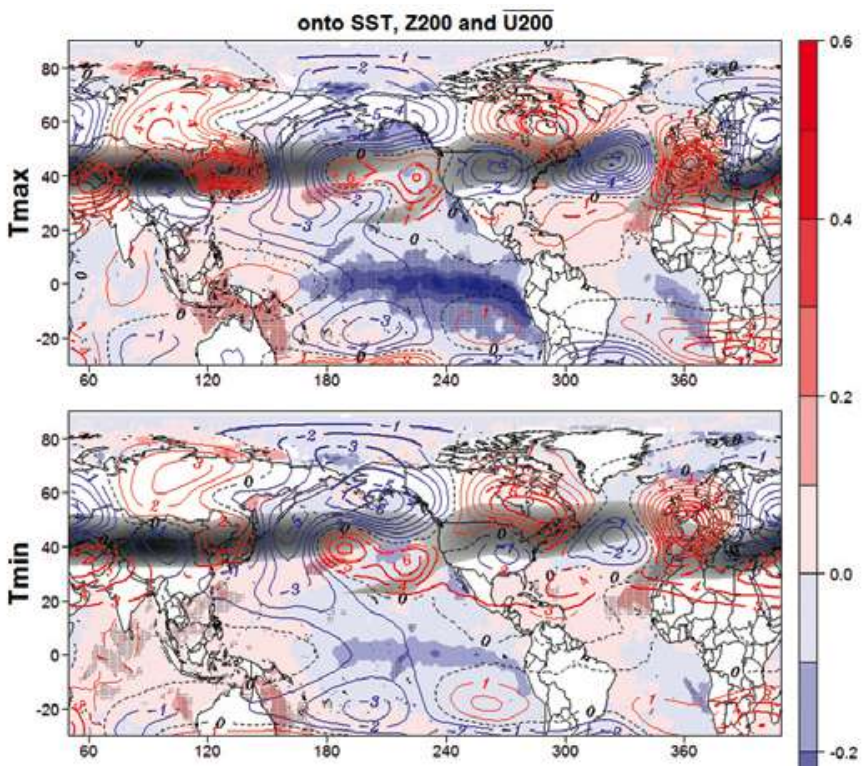

Fig. 2.- Mapas de regresión para JAS en 1952-1987 para Tmax (arriba) y Tmin (medio), y en 1957-2003 para Pcp (abajo), sobre SST $\left(^{\circ}\right.$, en colores; puntos negros para áreas estadísticamente significativas) y sobre Z200 (m, contornos; líneas azules para valores negativos, líneas rojas para los positivos, y líneas más gruesas para los contornos estadísticamente significativos.

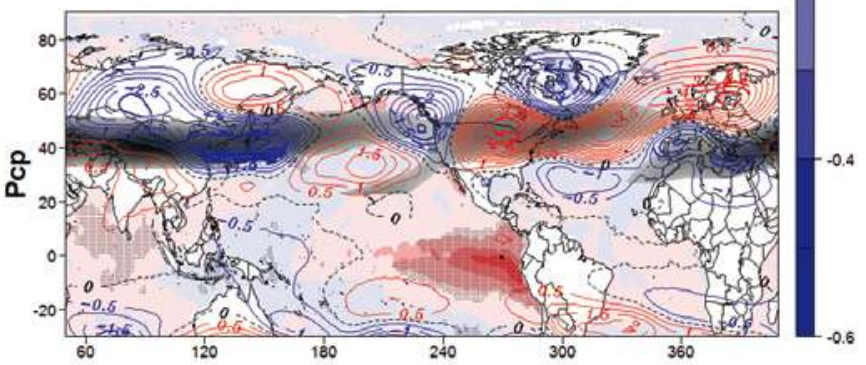
Cero en líneas discontinúas negras). La media de la U200 en gama de grises.
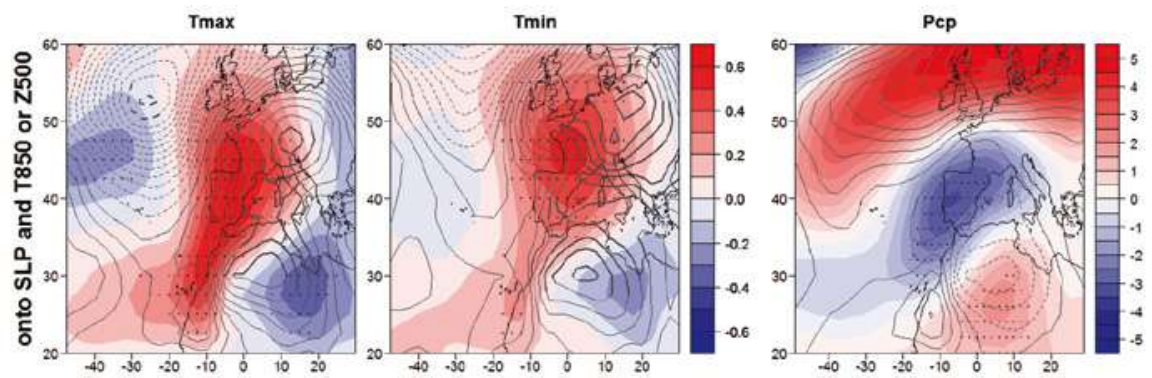

Fig. 3.- Mapas de regresión para JAS en 1952-1987 para Tmax (izquierda) y Tmin (medio), sobre T850 ( ${ }^{\circ}$, en colores; puntos negros para áreas estadísticamente significativas) y en 1957-2003 para Pcp (derecha) sobre Z500 (m, en colores; puntos negros para áreas estadísticamente significativas), y sobre SLP (hPa, contornos; líneas discontinuas para valores negativos y más gruesas para las estadísticamente significativas). 
Por lo tanto, la configuración regional descrita anteriormente como un aumento de la estabilidad meteorológica, viento del sur y aumento de la T850, explica el ascenso de Tmax y Tmin cuando aparece un patrón de La Niña, y lo contrario en el caso del patrón de El Niño. Pero también profundizaremos en el mecanismo de la teleconexión desde el Pacífico. En la configuración de Z200 (Fig. 2, arriba y medio, contornos) se muestra la típica respuesta de Gill (Gill, 1980) en el Pacífico Ecuatorial, alrededor de la anomalía en la SST, con dos centros de bajas (altas) presiones relativas al noroeste y al suroeste de la señal en el océano. En torno a los $40^{\circ} \mathrm{N}$ de latitud, se observa claramente una onda, atrapada en el jet (U200 en paleta de grises), con cinco centros de anomalías positivas y otros cinco de negativas (número de onda 5), es decir, una teleconexión tipo CGT. Estos resultados están en concordancia con los expuestos en Ding \& Wang (2005), Ding et al. (2011) y Xoplaki et al. (2003a, 2003b). En particular, Ding et al. (2011) describen dos tipos de respuesta; una para los veranos anteriores al invierno en el que se registra el pico máximo del fenómeno ENSO y la segunda correspondiente a los veranos posteriores al mismo. El primer caso se relaciona con una teleconexión tipo CGT. En nuestro estudio, para Tmax y Tmin, nos encontramos con un fenómeno de El Niño (La Niña) que acaba de comenzar a desarrollarse (Fig. 4), es decir en el verano anterior al invierno en el que se registrará el pico máximo del mismo. Por lo tanto, nuestros resultados coinciden con los de Ding et al. (2011).

Fig. 4.- Correlaciones en JAS entre Tmax (arriba), Tmin (medio) en 1952-87 y Pcp (abajo) en 1957-03 y los índices de El Niño; El Niño 1.2 (izquierda) y El Niño 4 (derecha), en las 24 posibles estaciones de 3 meses de los anteriores 2 años. La columna de más a la derecha de cada figura muestra la correlación de cada variable en el periodo y estación mencionada y los índices de El Niño en la misma estación y periodo ( $z$ en el eje $x$ y en la leyenda). La columna de más a la izquierda de cada

Niño 1.2

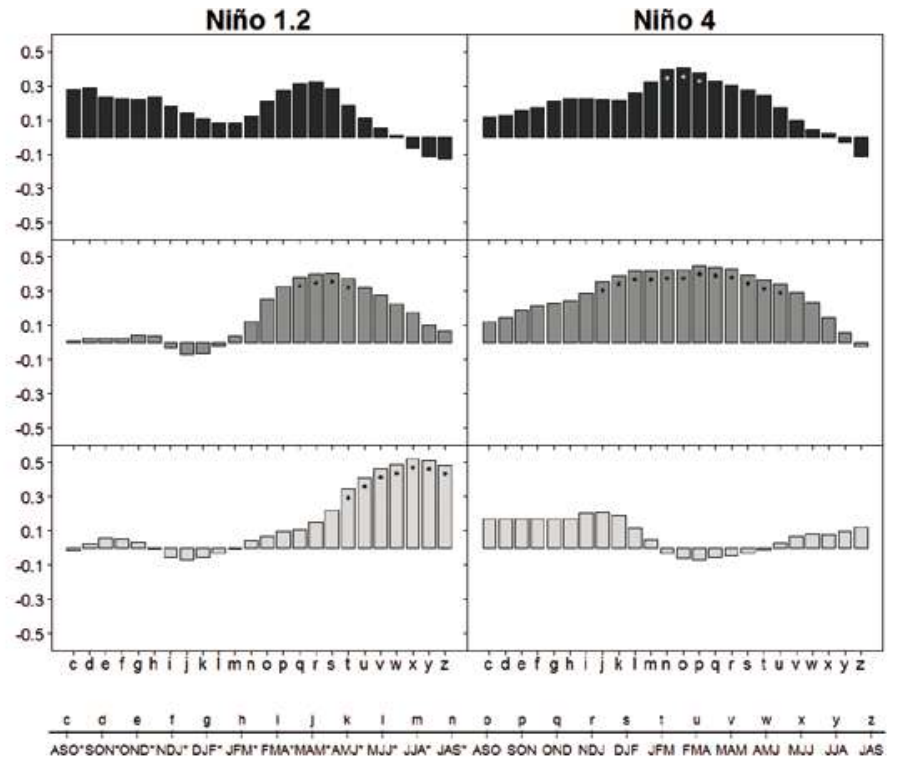
figura muestra la correlación entre cada variable en el periodo y estación mencionada y los índices de El Niño en agostoseptiembre-octubre (ASO) dos años antes (c en el eje x y en la leyenda), es decir en 1950-85 para Tmax y Tmin, y en 1955-01 para Pcp. Puntos blancos/negros para correlaciones estadísticamente significativas. En la leyenda el símbolo “"”" significa que la estación corresponde a entre un año y dos años antes. 
En el caso de la Pcp (Fig. 2, abajo), en el mapa de regresión sobre la SST aparece una configuración tipo El Niño (La Niña) del este, relacionado con un aumento (disminución) de la lluvia. Los valores de la proyección de la Pcp anómala y estandarizada sobre la SST anómala superan los $0,3^{\circ}$ en la zona este del Pacífico Ecuatorial, junto al continente. Analizando la configuración en Z200 (Fig. 2, abajo, contornos), encontramos un centro de bajas (altas) presiones significativas centrado en el noreste de la PI (incluyendo el PV), mientras que se aprecia un centro de altas (bajas) presiones relativas que se extienden por la parte norte del continente europeo. Esta situación atmosférica explica el aumento (disminución) de la inestabilidad meteorológica en la zona del PV, especialmente teniendo en cuenta que encontramos el reflejo en los diferentes niveles (Z500 y SLP; Fig. 3 , derecha) de esa presión relativamente baja (alta) a Z200. En el mapa de regresión sobre Z500 (Fig. 3, derecha, en colores), un centro de anomalías negativas (positivas) supera los $-3,5$ m y se sitúa en el oeste de la PI, lo que se traduce en un aumento (disminución) de la inestabilidad dinámica en el PV.
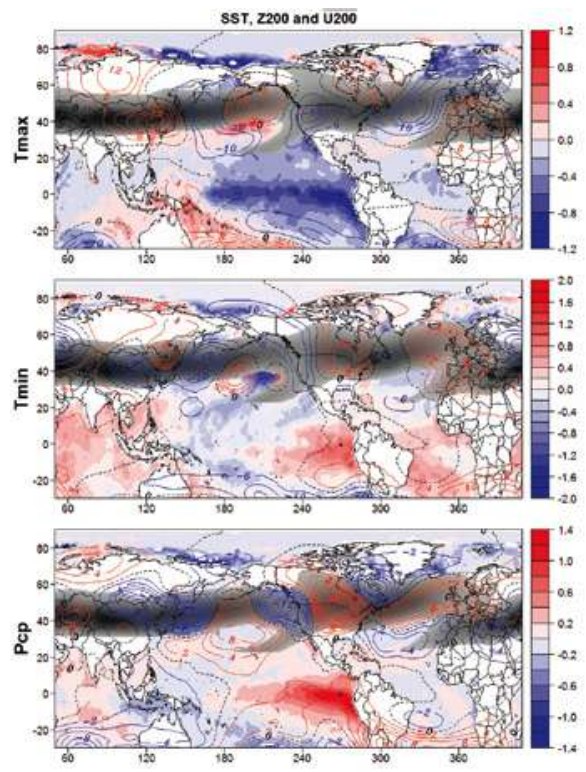
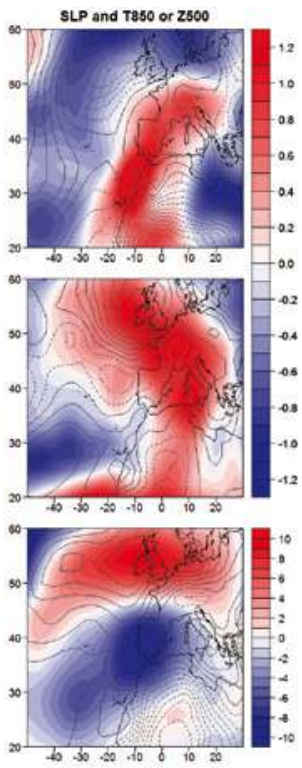

Fig. 5.- Compuesto positivo en JAS. Tmax (arriba), Tmin (medio) en 1952-87 para años por encima de 1 desviación estándar (std) y Pcp (abajo) en 1957-2003 para años por encima de 0,5 std. (izquierda) SST $\left({ }^{\circ}\right.$, en colores) y $\mathrm{Z} 200$ ( $\mathrm{m}$, en contornos, líneas azules para valores negativos, rojas para positivos y negra discontinua para cero). Media de U200 en paleta de grises. (derecha) T850 $\left({ }^{\circ}\right.$, en colores) para Tmax y Tmin (arriba y medio) y $\mathrm{Z} 500$ ( $\mathrm{m}$, en colores) para Pcp (abajo), y SLP (hPa, en contornos, líneas discontinuas para valores negativos).

En el mapa de regresión sobre la SLP (Fig. 3, derecha, contornos), encontramos un centro de bajas (altas) presiones en el norte de África que se extiende hasta abarcar toda la PI y también el PV. Por un lado, la presión relativamente baja en superficie (caso de El Niño), favorece que el flujo cálido y del sur atraviese el Mediterráneo aumentando la humedad que contiene y alcanzando el PV desde el noreste enfriándose y favoreciendo que se produzcan precipitaciones (aumento de la Pcp). La fuerte baroclinidad sobre el norte de África (anomalías positivas en Z500 sobre Marruecos) fomenta la advección 
mixta y cálida sobre Europa occidental. Por otro lado, la presión relativamente alta en superficie (caso de La Niña), favorece que el flujo relativamente cálido y seco atraviese la PI y alcance el PV con menor humedad, dificultando las precipitaciones (disminución de la Pcp). Esta situación atmosférica explica perfectamente el aumento de la Pcp sobre el PV en JAS cuando tenemos ENSO positivo en el este del Pacífico Ecuatorial, y un descenso de la Pcp sobre el PV en el caso de un ENSO negativo.

La onda que aparece en la configuración de Z200 (Fig. 2, abajo, contornos), se puede relacionar con un patrón en arco. En este caso, la teleconexión no está atrapada en el jet, como ocurría en el caso de temperatura, sino que aprovecha una debilidad del mismo para atravesarlo. Analizando la evolución de ENSO en los meses anteriores al verano (Fig. 4) podemos concluir que el pico máximo de El Niño (La Niña) se alcanzó en el invierno anterior, resultados que coinciden con los de Ding et al. (2011) que describían una teleconexión en arco en los veranos posteriores al pico de ENSO. Sin embargo, estos autores definían una teleconexión tipo PNA y en nuestro análisis encontramos una teleconexión más similar a la TNH (Mo \& Livezey, 1986). Según Barnston et al. (1991) El Niño (La Niña) produce la fase negativa (positiva) del patrón $\mathrm{TNH}$, el mismo que encontramos en este estudio. Además, considerando que la distribución espacial de las anomalías en la SST sugiere un Niño (Niña) del este (Fig. 2, abajo, en colores), la descripción de un patrón TNH también está en concordancia con los resultados de Yu et al. (2015).
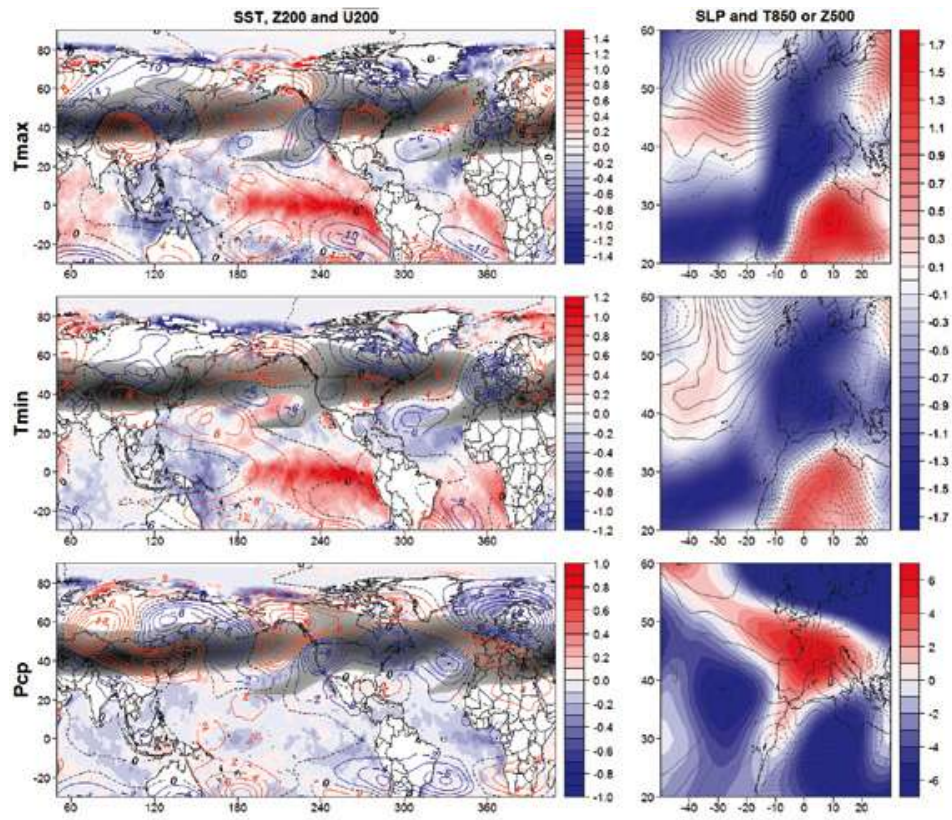

Fig. 6.-

Compuesto negativo en JAS. Igual que la Fig. 5

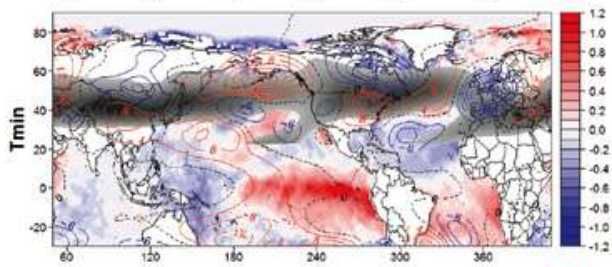
pero años por debajo de - 1 std para Tmax y Tmin, y años por debajo de $-0,5$ std para Pcp.

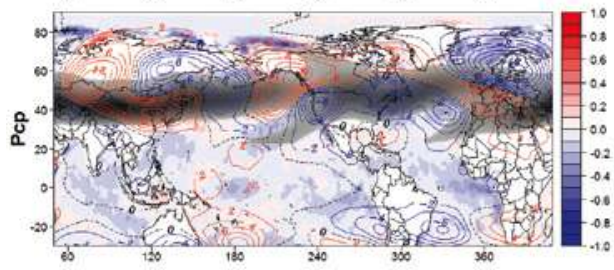


Hasta este punto se ha hecho un análisis lineal del fenómeno ENSO, asumiendo que los resultados para El Niño serán los contrarios que para La Niña. Pero de sobra es conocida la no linealidad de esta teleconexión con Europa, por lo que se calculan los mapas de compuestos positivos (Fig. 5) y negativos (Fig. 6) de Tmax, Tmin y Pcp para observar las señales en cada caso por separado. En el análisis de la Tmax (Fig. 5 y 6, arriba), los compuestos positivos muestran señal La Niña en todo el Pacífico Ecuatorial, mientras que en el compuesto negativo, aparece señal El Niño. En el mapa de Z200 aparece un patrón de teleconexión tipo CGT, con número de onda 5 , con signo opuesto en cada uno de los compuestos. Y en el mapa correspondiente al campo de T850, sobre el PV, aparecen anomalías positivas y negativas en los compuestos positivos y negativos respectivamente. Centrándonos en la SLP, en el compuesto positivo encontramos una configuración bastante poco definida, que favorece que las anomalías positivas en T850 produzcan una Tmax por encima de la media en el PV. Por otro lado, en el compuesto negativo, encontramos un claro flujo del continente sobre el área de estudio, que añadido a las anomalías negativas en el campo de T850, explican la Tmax por debajo de lo normal. Por lo tanto, la teleconexión entre ENSO y la Tmax en el PV podemos deducir que parece ser lineal.

En cambio, para la Tmin y la Pcp encontramos resultados distintos; la teleconexión parece estar sesgada a El Niño. Para los compuestos de la Tmin, mientras que en el negativo aparece señal ENSO, en el positivo no se muestra ningún patrón significativo en el Pacífico Ecuatorial. El compuesto negativo de la Tmin se relaciona con El Niño y aparece una teleconexión tipo CGT en el campo de Z200, similar a la del caso Tmax. Las anomalías negativas en T850 y el flujo del norte sobre el PV (Fig. 6, medio, derecha), explican una Tmin por debajo de lo normal. En el caso de los compuestos de la Pcp, el positivo presenta una señal del tipo El Niño del este en el Pacífico Ecuatorial. La dinámica de la teleconexión en este caso puede explicarse como un patrón tipo arco, concretamente la fase negativa de la TNH (Fig. 5, abajo, izquierda). En el campo de Z500, encontramos un centro de anomalías negativas en el sudeste de la PI, lo que favorece la inestabilidad y aumenta las probabilidades de precipitaciones convectivas en la zona del PV, fomentadas también por el flujo húmedo del océano en superficie (SLP).

\section{Conclusiones}

En este trabajo encontramos correlaciones estadísticamente significativas entre ENSO y la Tmax, Tmin y Pcp en el PV, en ciertas décadas y estaciones. Estas correlaciones son máximas en verano (JAS) en los periodos 1952-1987 para Tmax y Tmin y en 1957-2003 para la Pcp. Los valores mayores de correlación obtenidos se sitúan por debajo de los -0,8 para la Tmax, y los -0,7 para la Tmin. En el caso de la Pcp, las correlaciones son positivas y alcanzan los 0,7. Estas fuertes correlaciones dependen del área del Pacífico Ecuatorial que se estudie. Para la Tmax y la Tmin, las correlaciones significativas aparecen en los dos índices de El Niño analizados, pero las mayores se centran en El Niño 4, es decir, en el área central del Pacífico Ecuatorial. Sin embargo, en el caso de la Pcp, las correlaciones más fuertes aparecen en la zona más este de la cuenta, relacionadas con el índice de El Niño 1.2. Además, las correlaciones se mantienen altas y estadísticamente significativas si buscamos las relaciones de las variables con los índices 
de El Niño en estaciones anteriores. De manera que, alrededor de un año y medio antes siguen siendo estadísticamente significativas para la Tmax y la Tmin, y alrededor de medio año antes para la Pcp.

Centrándonos en el mecanismo que explica esta teleconexión, encontramos distintos resultados para las Tmax y Tmin, y para la Pcp. En el caso de la Tmax y la Tmin, una teleconexión tipo CGT parece explicar la relación entre un ENSO central y que va debilitándose, y la variabilidad en estas variables en el PV. Sin embargo, una teleconexión tipo $\mathrm{TNH}$, es la que parece ser responsable de la relación entre un ENSO del este que comienza a desarrollarse y la variabilidad de la Pcp en el PV.

Teniendo en cuenta la bien conocida no linealidad de la influencia de ENSO sobre Europa, analizamos al detalle la relación entre El Niño o La Niña por separado, y la variabilidad en el PV. Los resultados muestran que en el caso de la Tmax, la influencia de ENSO es lineal en verano (JAS), de manera que El Niño y La Niña presentan consecuencias opuestas en la variabilidad de esta variable en el PV. El Niño (La Niña) está relacionado con anomalías negativas (positivas) en la Tmax. Sin embargo, para la Tmin y la Pcp, no encontramos un comportamiento lineal. Los años con Tmin más fría de lo normal en la zona de estudio, se pueden relacionar con años en el que se produce El Niño, pero no parece existir relación entre los años con Tmin más cálida de lo normal y años en que se produce La Niña. Del mismo modo, los años más húmedos (mayor cantidad de Pcp) se pueden relacionar con El Niño, pero no los más secos (menor cantidad de Pcp) con La Niña.

Estos resultados podrían ayudar a mejorar las previsiones estacionales en la zona. Sin embargo, se deben realizar más estudios para probar estos resultados, como profundizar en el comportamiento de los extremos o verificar la utilidad de los mismos en un modelo de pronóstico estacional real.

\section{Referencias}

- Barnston, A. G., Livezey, R. E., \& Halpert, M. S. (1991). Modulation of Southern OscillationNorthern Hemisphere Mid-Winter Climate Relationships by the QBO. Journal of Climate. https:// doi.org/10.1175/1520-0442(1991)004<0203:MOSONH>2.0.CO;2

- Bergman, K. H. (1984). The climate of autumn 1983--featuring the conclusion of a major El Niño event. Monthly weather review.

- Biau, G., Zorita, E., Von Storch, H., \& Wackernagel, H. (1999). Estimation of precipitation by kriging in the EOF space of the sea level pressure field. Journal of Climate, 12(4), 1070-1085. https:// doi.org/10.1175/1520-0442(1999)012<1070:EOPBKI >2.0.CO;2

- Branstator, G. (2002). Circumglobal teleconnections, the jet stream waveguide, and the North Atlantic Oscillation. Journal of Climate, 15(14), 1893-1910. https://doi.org/10.1175/15200442(2002)015<1893:CTTJSW>2.0.CO;2

- Brönnimann, S. (2007). Impact of El Niño-Southern Oscillation on European climate. Rev. Geophys., 45(3), RG3003-. https://doi.org/10.1029/2006RG000199.1.INTRODUCTION

- Bulić, I. H., \& Kucharski, F. (2012). Delayed ENSO impact on spring precipitation over North/ Atlantic European region. Climate Dynamics, 38(11-12), 2593-2612. https://doi.org/10.1007/ s00382-011-1151-9 
- Capa-Morocho, M., Rodríguez-Fonseca, B., \& Ruiz-Ramos, M. (2014). Crop yield as a bioclimatic index of El Niño impact in Europe: Crop forecast implications. Agricultural and Forest Meteorology, 198-199, 42-52. https://doi.org/10.1016/j.agrformet.2014.07.012

- Cassou, C., \& Terray, L. (2001). Oceanic forcing of the wintertime low-frequency atmospheric variability in the North Atlantic European Sector: A study with the ARPEGE model. Journal of Climate, 14(22), 4266-4291. https://doi.org/10.1175/1520-0442(2001)014<4266:OFOTWL> 2.0.CO;2

- Davies, J. R., Rowell, D. P., \& Folland, C. K. (1997). North Atlantic and European seasonal predictability using an ensemble of multidecadal atmospheric GCM simulations. International Journal of ..., 17, 1263-1284. https://doi.org/10.1002/(SICI)1097-0088(199710)17:12<1263::AIDJOC191>3.0.CO;2-1

- Ding, Q., \& Wang, B. (2005). Circumglobal teleconnection in the Northern Hemisphere summer. Journal of Climate, 18(17), 3483-3505. https://doi.org/10.1175/JCLI3473.1

- Ding, Q., Wang, B., Wallace, J. M., \& Branstator, G. (2011). Tropical-extratropical teleconnections in boreal summer: Observed interannual variability. Journal of Climate, 24(7), 1878-1896. https:// doi.org/10.1175/2011JCLI3621.1

- Dommenget, D., Bayr, T., \& Frauen, C. (2013). Analysis of the non-linearity in the pattern and time evolution of El Niño southern oscillation. Climate Dynamics, 40(11-12), 2825-2847. https:// doi.org/10.1007/s00382-012-1475-0

- Fraedrich, K. (1994). An ENSO impact on Europe? Tellus A. https://doi.org/10.1034/j.16000870.1994.00015.x

- Gill, A. E. (1980). Some simple solutions for heat induced tropical circulation. Quarterly Journal of the Royal Meteorological Society. https://doi.org/10.1002/qj.49710644905

- Gouirand, I., \& Moron, V. (2003). Variability of the impact of El Niño-Southern Oscillation of sealevel pressure anomalies over the North Atlantic in January to March (1874-1996). International Journal of Climatology, 23(13), 1549-1566. https://doi.org/10.1002/joc.963

- Hannachi, A. (2001). Toward a nonlinear identification of the atmospheric response to ENSO. Journal of Climate, 14(9), 2138-2149. https://doi.org/10.1175/1520-0442(2001)014<2138:TAN IOT $>2.0 . C O ; 2$

- Herrera, S., Gutiérrez, J. M., Ancell, R., Pons, M. R., Frías, M. D., \& Fernández, J. (2012). Development and analysis of a 50-year high-resolution daily gridded precipitation dataset over Spain (Spain02). International Journal of Climatology, 32(1), 74-85. https://doi.org/10.1002/ joc. 2256

- Kalnay, E., Kanamitsu, M., Kistler, R., Collins, W., Deaven, D., Gandin, L., ... Joseph, D. (1996). The NCEP/NCAR 40-year reanalysis project. Bulletin of the American Meteorological Society. https:// doi.org/10.1175/1520-0477(1996)077<0437:TNYRP>2.0.CO;2

- Kiladis, G. N., \& Diaz, H. F. (1989). Global Climatic Anomalies Associated with Extremes in the Southern Oscillation. Journal of Climate. https://doi.org/10.1175/15200442(1989)002<1069:GCAAWE >2.0.CO;2

- López-Parages, J., \& Rodríguez-Fonseca, B. (2012). Multidecadal modulation of El Niño influence on the Euro-Mediterranean rainfall. Geophysical Research Letters, 39(2), 1-7. https://doi. org/10.1029/2011GL050049

- López-Parages, J., Rodríguez-Fonseca, B., Dommenget, D., \& Frauen, C. (2016). ENSO influence on the North Atlantic European climate: a non-linear and non-stationary approach. Climate Dynamics, 47(7-8), 2071-2084. https://doi.org/10.1007/s00382-015-2951-0

- López-Parages, J., Rodríguez-Fonseca, B., \& Terray, L. (2015). A mechanism for the multidecadal modulation of ENSO teleconnection with Europe. Climate Dynamics, 45(3-4), 867-880. https://doi. org/10.1007/s00382-014-2319-x 
- Mathieu, P. P., Sutton, R. T., Dong, B., \& Collins, M. (2004). Predictability of winter climate over the North Atlantic European region during ENSO events. Journal of Climate, 17(10), 1953-1974. https://doi.org/10.1175/1520-0442(2004)017<1953:POWCOT>2.0.CO;2

- Mills, G. F. (1995). Principal Component Analysis of precipitation and rainfall regionalization in Spain. Theoretical and Applied Climatology, 50(3-4), 169-183. https://doi.org/10.1007/ BF00866115

- Mo, K. C., \& Livezey, R. E. (1986). Tropical-Extratropical Geopotential Height Teleconnections during the Northern Hemisphere Winter. Monthly Weather Review. https://doi.org/10.1175/15200493(1986)114<2488:TEGHTD>2.0.CO;2

- Pozo-Vázquez, D., Esteban-Parra, M. J., Rodrigo, F. S., \& Castro-Díez, Y. (2001). The Association between ENSO and Winter Atmospheric Circulation and Temperature in the North Atlantic Region. Journal of Climate, 14(16), 3408-3420. https://doi.org/10.1175/1520-0442(2001)014<3408:TABEA $\mathrm{W}>$ 2.0.CO;2

- Raible, C. C., Luksch, U., \& Fraedrich, K. (2003). Precipitation and Northern Hemisphere regimes. Atmospheric Science Letters, 5(1-4), 43-55. https://doi.org/10.1016/j.atmoscilet.2003.12.001

- Rayner, N. A., Parker, D. E., Horton, E. B., Folland, C. K., Alexander, L. V., \& Rowell, D. P. (2003). Global analyses of sea surface temperature, sea ice, and night marine air temperature since the late nineteenth century. Journal of Geophysical Research. https://doi.org/10.1029/2002JD002670

- Sáenz, J., Zubillaga, J., \& Rodríguez-Puebla, C. (2001). Interannual winter temperature variability in the north of the Iberian Peninsula. Climate Research, 16(3), 169-179. https:/doi.org/10.3354/ cr016169

- Serrano, A., García, J. A., Mateos, V. L., Cancillo, M. L., \& Garrido, J. (1999). Monthly modes of variation of precipitation over the Iberian Peninsula. Journal of Climate, 12(9), 2894-2919. https:// doi.org/10.1175/1520-0442(1999)012<2894:MMOVOP >2.0.CO;2

- Stockdale, T. N., Anderson, D. L. T., Alves, J. O. S., \& Balmaseda, M. A. (1998). Global seasonal rainfall forecasts using a coupled ocean- atmosphere model. Nature.

- Sutton, R. T., \& Hodson, D. L. R. (2003). Influence of the ocean on North Atlantic climate variability 1871-1999. Journal of Climate, 16(20), 3296-3313. https://doi.org/10.1175/15200442(2003)016<3296:IOTOON>2.0.CO;2

- Toniazzo, T., \& Scaife, A. A. (2006). The influence of ENSO on winter North Atlantic climate. Geophysical Research Letters, 33(24), 1-5. https://doi.org/10.1029/2006GL027881

- Wu, A., \& Hsieh, W. W. (2004a). The nonlinear association between ENSO and the Euro-Atlantic winter sea level pressure. Climate Dynamics, 23(7-8), 859-868. https://doi.org/10.1007/s00382004-0470-5

- Wu, A., \& Hsieh, W. W. (2004b). The nonlinear Northern Hemisphere winter atmospheric response to ENSO. Geophysical Research Letters, 31(2), 2-5. https://doi.org/10.1029/2003GL018885

- Xoplaki, E., González-Rouco, J. F., Luterbacher, J., \& Wanner, H. (2003a). Mediterranean summer air temperature variability and its connection to the large-scale atmospheric circulation and SSTs. Climate Dynamics, 20(7-8), 723-739. https://doi.org/10.1007/s00382-003-0304-x

- Xoplaki, E., González-Rouco, J., Gyalistras, D., Luterbacher, J., Rickli, R., \& Wanner, H. (2003b). Interannual summer air temperature variability over Greece and its connection to the large-scale atmospheric circulation and Mediterranean SSTs 1950-1999. Climate Dynamics, 20(5), 537-554. https://doi.org/10.1007/s00382-002-0291-3

- Yu, B., Zhang, X., Lin, H., \& Yu, J. Y. (2015). Comparison of Wintertime North American Climate Impacts Associated with Multiple ENSO Indices. Atmosphere - Ocean, 53(4), 426-445. https://doi. org/10.1080/07055900.2015.1079697 
- Zanchettin, D., Franks, S. W., Traverso, P., \& Tomasino, M. (2008). On ENSO impacts on European wintertime rainfalls and their modulation by the NAO and the Pacific multi-decadal variability described through the PDO index. International Journal of Climatology. https:/doi.org/10.1002/ joc.1601

- Zhang, L., Wang, C., \& Wu, L. (2012). Low-frequency modulation of the Atlantic warm pool by the Atlantic multidecadal oscillation. Climate Dynamics, 39(7-8), 1661-1671. https://doi.org/10.1007/ s00382-011-1257-0

- Zhang, W., Wang, Z., Stuecker, M. F., Turner, A. G., Jin, F.-F., \& Geng, X. (2018). Impact of ENSO longitudinal position on teleconnections to the NAO. Climate Dynamics, 0(0), 1-18. https://doi. org/10.1007/s00382-018-4135-1

- Zorita, E., Kharin, V., \& Vonstorch, H. (1992). The Atmospheric Circulation and Sea-Surface Temperature in the North-Atlantic Area in Winter - Their Interaction and Relevance for Iberian Precipitation. Journal of Climate. https://doi.org/Doi 10.1175/1520-0442(1992)005<1097:Tacass> 2.0.Co;2 